\title{
Evolution of local adaptations in dispersal strategies
}

\author{
Andreas Gros ${ }^{1}$, Hans Joachim Poethke ${ }^{2}$ and Thomas Hovestadt ${ }^{3}$ \\ University of Würzburg, Field Station Fabrikschleichach, \\ Glashüttenstrasse 5, 96181 Rauhenebrach, Germany
}

March 14, 2006

${ }^{1}$ andreas.gros@biozentrum.uni-wuerzburg.de

${ }^{2}$ poethke@biozentrum.uni-wuerzburg.de

${ }^{3}$ hovestadt@biozentrum.uni-wuerzburg.de 
The optimal probability and distance of dispersal largely depend on the risk to end up 3 in unsuitable habitat. This risk is highest close to the habitat's edge and consequently, 4 optimal dispersal probability and distance should decline towards the habitat's border. 5 This selection should lead to the emergence of spatial gradients in dispersal strategies. 6 However, gene flow caused by dispersal itself is counteracting local adaptation. Using 7 an individual based model we investigate the evolution of local adaptations of dispersal 8 probability and distance within a single, circular, habitat patch. We compare evolved 9 dispersal probabilities and distances for six different dispersal kernels (two negative ex10 ponential kernels, two skewed kernels, nearest neighbour dispersal and global dispersal) 11 in patches of different size. For all kernels a positive correlation between patch size and 12 dispersal probability emerges. However, a minimum patch size is necessary to allow for 13 local adaptation of dispersal strategies within patches. Beyond this minimum patch area 14 the difference in mean dispersal distance between center and edge increases linearly with 15 patch radius, but the intensity of local adaptation depends on the dispersal kernel. Except for global and nearest neighbour dispersal, the evolved spatial pattern are qualitatively similar for both, mean dispersal probability and distance. We conclude, that inspite of the gene-flow originating from dispersal local adaptation of dispersal strategies is possible if a habitat is of sufficient size. This presumably holds for any realistic type of dispersal kernel.

Keywords: dispersal distance, dispersal probability, dispersal kernel, spatial pattern, individual-based model 


\section{${ }_{23} 1$ Introduction}

${ }_{24}$ The evolution of dispersal strategies has become an important topic in theoretical ecology

25 (e.g. Hamilton and May 1977; Comins et al. 1980; Hovestadt et al. 2001; Hanski et al.

26 2004). From an individual's perspective dispersal may be motivated by a number of

${ }_{27}$ reasons, e.g. avoiding competition for resources (either inter- or intraspecific; Lambin

et al. 2001), minimising kin competition (e.g. Hamilton and May 1977; Comins 1982; Kisdi 2004), avoiding inbreeding (e.g. Motro 1991) or coping with the temporal variability of resource availabilities (Levin et al. 1984; Travis and Dytham 1999). Generally, individuals should disperse as long as they expect a higher fitness away from their natal habitat (Frank 1986; Metz and Gyllenberg 2001; Poethke and Hovestadt 2002; Dytham 2003). However, dispersal is associated with costs and depending on landscape configuration dispersal may carry a substantial risk: as long as habitat is abundant, dispersal is not very risky, but when suitable patches become scarce and widely spread, dispersers are likely to end up in unsuitable habitat: the matrix (Hastings 1983; Travis and Dytham 1999). Consequently, we can predict the adaptation of dispersal strategies to general landscape characteristics (Hovestadt et al. 2001).

In natural landscapes, the quality, density and distribution of habitat is typically variable across space. Obviously, the optimal dispersal strategy will depend on the actual position within a heterogeneous landscape. Selection should therefore favour local adaptation of dispersal strategies, at least as long as offspring disperses into a similar spatial context. However, dispersal is also the factor responsible for gene flow and fundamentally operating against local adaptation (Haldane 1956; Case and Taper 2000). The question then arises under which conditions spatial patterns in dispersal strategies can evolve.

In this paper we use an individual-based model to investigate the evolution of dispersal probability and dispersal distance of asexual annual plants within a single circular patch located in a hostile matrix. In contrast to Hamilton and May (1977), Comins et al. (1980), and Rousset and Gandon (2002), who implement dipsersal costs as an external factor, in 


\section{${ }_{67} 2$ Material and Methods}

68 one type of dispersal kernel. by Travis and Dytham (1999).

our model the structure of the landscape determines the costs of dispersal due to the loss of offspring dispersing into the matrix (Hovestadt et al. 2001). The evolutionary pressure is mainly exerted by the risk to disperse offspring across the edge of a habitat. This should select for decreased dispersal probability and dispersal distance in small patches or in large patches near the border to the matrix. In the latter case however, gene flow may counteract selection. Therefore, population size and accessibility of habitat should play a crucial role in the emergence of locally adapted dispersal strategies. The topic has already been addressed by Travis and Dytham (1999), who demonstrated that local adaptation of dispersal strategies can emerge in a complex landscape. However, their model consisted of demes with local, non-spatial population dynamics. In addition, Travis and Dytham (1999) did not aim at a systematic investigation of the effect of patch respectively population size on the evolution of dispersal distance and was limited to only

Our goal is to understand under which conditions the emergence of locally adapted dispersal strategies becomes possible and to compare the evolutionary outcome for different dispersal kernels in a very simple landscape, which separates our study from the one (Berec 2002) of square landscape cells which can be either habitat or matrix. Each habitat cell can support only one adult plant which is placed in the cell's center. Therefore the spatial scale depends on the plant's size. Within an unbounded world of matrix cells we placed a single circular habitat patch of variable radius $(25,50,75,100,125$ and 150 cells) (Figure 1). We use six dispersal kernels in this study: two negative exponential kernels $(\mathrm{NE}$ and $\mathrm{NE}+$ ), two skewed kernels $(\mathrm{S}$ and $\mathrm{S}+$ ) as well as nearest neighbour $(\mathrm{NN})$ and global dispersal $(\mathrm{G})$ (see below and Table 1). 
Each time step of the model represents a reproduction cycle of annual parthenogenetic plants. Three different procedures are executed during each time step, i.e. (i) reproduction, (ii) dispersal and (iii) post-dispersal competition.

(i) Reproduction: For simplicity, density-independent mortality of seeds or seedlings is not explicitly modelled. Thus each plant is considered to be a point source for seedlings rather than seeds. Each mother produces a constant number of five $(m=5)$ descendants. Each descendant is characterised by two continuous genetic characters $G_{d}$ and $G_{p} . G_{p}$ is coding for the propensity to disperse, whereas $G_{d}$ codes for the mean dispersal distance. For simulation experiments with kernels characterised by dispersal distance only ( $\mathrm{S}$ and $\mathrm{NE}$, see below), $G_{p}$ is without function and consequently selectively neutral. The same holds for $G_{d}$ in simulation experiments with kernels G and NN, which are only defined by a dispersal probability.

Seedlings inherit the genes for $G_{d}$ and $G_{p}$ from their mother but genes mutate with a probability of 0.001 during this process. In case of a mutation $G_{d}$ is altered by multiplying its value with a random value from the uniform interval $[0.9,1.1]$. This keeps $G_{d}$ positive and creates mutation steps proportional to the value of the genetic character. $G_{p}$ is altered by adding a random value from the uniform interval $[-0.025,0.025]$. Its values are kept within the interval $[0,1]$ by truncation. In each simulation run the values of $G_{d}$ and $G_{p}$ in the start population are uniformly distributed within the interval $[0,200]$ and $[0,1]$ respectively.

(ii) Dispersal: During the dispersal process all offspring are distributed according to their parent's dispersal strategy (dispersal distance $\left(G_{d}\right)$ and/or dispersal probability $\left.\left(G_{p}\right)\right)$. Whenever a dispersing offspring ends up in a matrix cell, it dies. There are no energetic or allocation costs associated with the decision to disperse $\left(G_{p}\right)$ or a specific mean dispersal distance $G_{d}$, i.e., the cost of dispersing seeds over a long distance is similar to short distance dispersal.

In our experiments we compare the performance of six frequently used dispersal 
kernels (see Table 1 for a pictographic compilation):

1. Global $(\mathrm{G})$ : The destination cell of a disperser is drawn randomly from all possible landscape cells. This kernel resembles the island model of Hamilton and May (1977) with the modification, that our landscape is heterogeneous. Only the dispersal probability $\left(G_{p}\right)$ is subject to evolution. In contrast to the other kernels this kernel requires a bounded landscape. Therefore, we restrict the dimension of the landscape to $400 \mathrm{X} 400$ cells in all simulation experiments implementing this kernel. The chance of a dispersing individual to arrive in a habitat cell is determined by the number of habitat cells compared to the total number of cells (equals $p$ of Hamilton and May 1977).

2. Nearest Neighbour (NN): A disperser draws one of the eight adjacent cells at random as destination (Travis and Dytham 1999). A similar kernel is suggested by Comins (1982) for dispersal between discrete colonies of animals living on a patchy resource, and seed and pollen distributions in continuous populations of plants. Because the dispersal distance is fixed, only the propensity to disperse $\left(G_{p}\right)$ evolves.

3. Negative-exponential (NE): A disperser draws a direction and distance at random. The dispersal distance is taken from a negative-exponential probability density function (1) with mean $G_{d}=1 / \alpha$.

$$
f(x)=\alpha e^{-\alpha x}
$$

Dispersal probability is dependent on dispersal distance in this kernel and only the dispersal distance $\left(G_{d}\right)$ is subject to evolution. As distance is measured from the center of a cell, a seedling remains philopatric if the dispersal distance is short enough to deposit the seedling within the maternal cell. Levin et al. (2003) states that this is probably the most common dispersal kernel in models (e.g. Murrell et al. 2002; Streiff et al. 1999; Yao et al. 1999; Bullock and Clarke 
2000; Hovestadt et al. 2000; Nathan et al. 2000; Bleher et al. 2002) and does properly fit seed distributions in dense aggregations of plants like forests.

4. Negative-exponential plus dispersal probability $(\mathrm{NE}+)$ : In this kernel the calculation of dispersal distances follows the same rules as in kernel NE, except that a dispersal move always starts at the maternal cell's border, to ensure that any dispersing seedling will fall into a cell different from its origin. The starting point of a dispersal move is the intersection point of a randomly chosen direction and the maternal cell's border. Hence, $G_{d}$ (as a parameter of Equation 1) in this kernel is the mean dispersal distance from the maternal cell's border. In contrast to kernel NE, a seedling disperses only with probability $G_{p}$. Both genetic characters, $G_{p}$ and $G_{d}$, evolve.

5. Skewed (S): Dispersal distances are drawn from a right-skewed probability density function (2) with mean $G_{d}=2 / \alpha$, following Hanski (1994) and Appelt and Poethke (1997):

$$
p(x)=\alpha^{2} x e^{-\alpha x}
$$

By having its peak away from the source this kernel predicts more seeds to travel beyond the immidiate influence of the mother plant than with the negative exponential kernels. In contrast to leptokurtic distributions, like the "2Dt" kernel suggested by Clark et al. (1999), it is determinded by just one parameter $(\alpha)$, and is not 'fat-tailed'. Like with kernel NE only the dispersal distance, determined by the genetic character $G_{d}$, undergoes evolutionary changes and seeds that do not cross the maternal cell's border, stay philopatric.

6. Skewed plus dispersal probability $(\mathrm{S}+)$ : The process determining whether or not to disperse is the same as in kernel $\mathrm{NE}+$ and like with kernel $(\mathrm{NE}+)$ a dispersal move starts at a cell's border. The calculation of dispersal distances complies the same distribution as in kernel S (Equation 2). Both genetic char- 


\section{${ }_{175} 3$ Results}

(iii) Post-dispersal competition: After their offspring's dispersal, all adult plants die. For each cell a single seedling is randomly drawn among all its immigrants to establish and reproduce in the next time-step. All other seedlings die, i.e. we do not consider the establishment of a seed bank. To test whether local adaptation has evolved in $G_{p}$ and $G_{d}$, we separate the habitat into four areas: a circular area in the center (A1) and three consecutive rings (A2-A4) (other modes of partitioning do not alter the results presented in the following). Each of these areas includes an approximately equal number of cells. This implies that the rings get "thinner" towards the edge of the habitat (Figure 1).

The number of repeated simulation runs conducted is inversely proportional to the patch's area. For a radius of 150 cells (70685 habitat cells in total) we only run one simulation, while for patches with a radius of 25 cells, 36 replicate simulation runs $(36 \times 1963$ habitat cells $=70668$ habitat cells in total). Hence, the evolutionary process in each landscape configuration is being evaluated on the basis of about an equal number of habitat cells. Simulations with one evolving trait ran for 50000, for those with two traits 100000 time steps were simulated. We set longer simulation times for scenarios with two traits because multi-dimensional evolutionary trajectories towards equilibrium usually take longer time. The small number of replicates in large habitats was determined by the substantial computation time needed. Mean values of $G_{d}$ and $G_{p}$ were averaged over all replicates of simulations of a landscape-kernel-combination.

We first consider the evolution of the dispersal probability, i.e. the fraction of seedlings deposited outside the cell of origin. Dispersal probabilities depend on both, the size of the habitat patch and the dispersal kernel. Dispersal probability is by far the lowest 
with global dispersal $(\mathrm{G})$ but also shows the strongest increase with habitat patch size (Figure 2a). Both exponential kernels $(\mathrm{NE}$ and $\mathrm{NE}+$ ) and the skewed kernels ( $\mathrm{S}$ and $\mathrm{S}+$ ) also exhibit an increase in dispersal probability with increasing patch size while nearest neighbour dispersal (NN) is hardly sensitive to patch area. This difference in response to patch size results in a decreasing difference in dispersal probabilities between different kernels for increasing patch area. For small patches with a radius of 25 cells only $20 \%$ of the seeds are dispersed outside the mother's cell with kernel G compared to more than 70 $\%$ with kernel $\mathrm{NE}$ and about $90 \%$ with kernel $\mathrm{NE}+, \mathrm{S}$ and $\mathrm{S}+$. For patches with a radius of 150 cells evolutionarily stable dispersal probabilities are $50 \%$ for global dispersers and nearly $100 \%$ for kernels $\mathrm{S}, \mathrm{S}+$ and $\mathrm{NE}+$. A variance of less than 0.006 in the mean dispersal probabilities across simulation runs even in the smallest patches indicates that results are not strongly affected by random effects.

Mean dispersal distance is not an evolvable trait for either nearest neighbour dispersal $(\mathrm{NN})$ or global dispersal $(\mathrm{G})$. For kernel $\mathrm{NN}$ it is fixed to the mean distance between neighbouring cells $(=1.21)$ and for global dispersal to approximately 200 cells, i.e. half the dimension of the simulated landscape. For the two negative-exponential kernels (NE and $\mathrm{NE}+$ ) as well as the skewed kernels $(\mathrm{S}$ and $\mathrm{S}+$ ) evolved mean dispersal distances strongly respond to the size of the habitat patch. Even for small patches they are much smaller than the patch radius. While evolved dispersal probabilities are higher for the $\mathrm{NE}+$ kernel, highest distances evolve with the NE kernel (Figure 2b). This contrasts to the skewed kernels $\mathrm{S}$ and $\mathrm{S}+$, where hardly any difference in mean dispersal distances evolves between the two kernels. Furthermore, the mean dispersal distances of kernels $\mathrm{S}+$ and $\mathrm{S}$ are about the same as with kernel $\mathrm{NE}+($ Figure $2 \mathrm{~b}$ ) in all patch sizes. To investigate the influence of kin-competition we alter the number of individual offspring from $m=5$ to either $m=2$ or $m=10$. With $m=2$ the evolving dispersal probabilities respectively distances drop in all kernels, whereas $m=10$ leads to an increase in the values of both genetic characters.

We further check for the emergence of local adaptation in the distribution of dispersal 
207 parameters $\left(G_{p}\right.$ and $\left.G_{d}\right)$ for each of the dispersal kernels. Obviously, there is no spatial

correlation between the mother's cell and the target cell of dispersed seedlings for global dispersal. Consequently, no local adaptation can evolve for this dispersal kernel. For the other five kernels $(\mathrm{NN}, \mathrm{NE}, \mathrm{NE}+, \mathrm{S}$ and $\mathrm{S}+$ ) the development of a spatial structure critically depends on patch size. While we find no spatial structure in the experiments with small habitat patches a pronounced structure evolves in the largest patches (Figures 3 and 4$)$. Mean dispersal probability $\left(\overline{G_{p}}\right)$ as well as mean dispersal distance $\left(\overline{G_{d}}\right)$ of individuals is always higher in the centre of the patches (area A1) than at the perimeter (area A4). This difference is strongly dependent on patch size (Figure 5) and may exceed $30 \%$ in large patches.

To control for the effect of mutation rate we run simulations with either a tenfold mutation rate or no mutations at all, but this has very little effect on our results. Only in simulations where evolved values of $G_{p}$ are close to 1 the results of simulations with no mutations stay slightly below the values achieved with a mutation rate of 0.001 or 0.01 .

\section{Discussion}

Our simulations clearly demonstrate the dual effect of patch size and dispersal mode on the evolution of dispersal strategies. Depending on the dispersal kernel, different evolutionary adjustments are possible: either by changes in the propensity to disperse, in mean dispersal distance, or both. The evolving strategies are a result of a basic tradeoff between reducing the loss of offspring dispersing into the matrix (Comins et al. 1980) and the possibility to escape kin-competition (Hamilton and May 1977; Ezoe 1998; Kasuya 2000; Kisdi 2004). Other factors favouring dispersal are of minor importance in our model because it lacks any environmental stochasticity.

For any dispersal kernel, the risk of losing offspring into the surrounding matrix will always decline as patch size increases. Consequently we find a consistent increase in dispersal probabilities with increasing patch area in all dispersal kernels except NN. The 
risk of loosing offspring into the matrix also depends on the dispersal distance and is largest for the kernels with the largest mean dispersal distance. This argument can explain why with global dispersal, which has the highest mean dispersal distance, the lowest dispersal probabilities evolved, and why this kernel is most sensitive to changes in patch size. With respect to dispersal distance the order of kernels is NN, with a dispersal distance of only one cell, followed by NE $+, \mathrm{S}, \mathrm{S}+$ (evolving similar dispersal distances), NE and G. However, considering dispersal probability, the inverse order emerges (except for kernel $\mathrm{NN}$ ): $\mathrm{G}, \mathrm{NE}, \mathrm{NN}$, followed by $\mathrm{NE}+, \mathrm{S}$ and $\mathrm{S}+$. It must be noted that the dispersal probability for kernels NE and S is not an evolvable trait as such but the emergent result of the evolution on mean dispersal distance $\left(\overline{G_{d}}\right)$. It should also be noted that the highest dispersal probabilities evolve in the only kernels with two parameters $(\mathrm{NE}+$ and $\mathrm{S}+$ ), i.e. the kernels which allow the most flexible changes in dispersal strategy.

The results with kernel NN (nearest neighbour) do not fit into this general view. Even though mean dispersal distance is the lowest with this kernel, evolved dispersal probabilities are lower than with kernel $\mathrm{NE}+$ and are hardly sensitive to changes in patch area. Presumably, this is a consequence of the restricted ability to escape strong kincompetition if dispersal is exclusively limited to the neighbouring cells (cf. Comins et al. 1980). This is confirmed by the effect of altering the number of individual offspring. When $m$ is reduced to 2, kin-competition for free space declines and consequently, the evolving dispersal probabilities respectively distances drop in all kernels. In contrast, with $m=10$ the increase in kin-competition leads to an increase in the values of both genetic characters. For both the exponential and the skewed kernels, mean dispersal distance also increases as patches become larger. It is striking that the mean values of $G_{d}$ with kernel $\mathrm{NE}$ are higher than those for kernel $\mathrm{NE}+$ (compare Figures $4 \mathrm{a}$ and $4 \mathrm{~b})$. This is caused by the evolutionary "challenge" to regulate philopatry and dispersal distances by a single parameter in kernel $\mathrm{NE}$, while $\mathrm{NE}+$ allows a separate regulation of these two traits. To achieve a similar dispersal probability as with kernel $\mathrm{NE}+$, individuals utilizing kernel $\mathrm{NE}$ need to increase mean dispersal distance, as otherwise too many progeny would remain 
within the natal cell and suffer from higher kin-competition. In contrast, the skewed kernel $\mathrm{S}$ evolves the same dispersal distances as $\mathrm{S}+$. Having its peak away from the source leaving the natal cell with kernel $\mathrm{S}$ is more likely than with NE, even if mean dispersal distance is lower.

The dispersal kernels differ quite remarkably in their tendency to develop spatial patterns in dispersal probability or distance. Obviously, local adaptation cannot and did not evolve with global dispersal as the starting position of a dispersing individual has no influence on the target position it will eventually reach. Surprisingly, we also found little evidence for the evolution of local adaptation under kernel NN, even though we observed some decline in parameter $G_{p}$ in the very outermost ring of cells. The very low dispersal distance of this kernel rather seems to promote the emergence of spatially distinct clusters of individuals of common descent (cf. Figure 6), a pattern frequently detected in such kinds of grid based simulations (e.g. Tilman et al. 1997; Levin and Pacala 1997; Ennos 2001). Travis and Dytham (1999) also implemented kernel NN in their simulation study to investigate local adaptation in fractal landscapes. They found a much broader range for the evolved dispersal probabilities than we and describe a clear selection for dispersal inside a patch and a distinct evolution towards non-dispersal at the habitat border. This is most likely caused by considering patches consisting of demes with local population dynamics each rather than cells consisting of only one individual. The higher capacities of surrounding cells excert less pressure against dispersal in the inner areas of a habitat by granting a higher probability of settling for immigrants. The clear selection for non-dispersal at the edge of their patches may be caused by the difficulty of gene flow introduced by the "rugged" shape of fractal patches.

For the two exponential kernels and the skewed kernels - which are probably closest to naturally occurring kernels - we observe the development of a weak spatial gradient for both dispersal probability and especially dispersal distance in patches with a radius of 50. From then on, the difference in mean dispersal distance between the centre of the patch (A1) and the outermost ring (A4) increases rather linearly (Figure 5b). In the 
largest patch mean dispersal distance is about one third larger in the centre than in the edge of the patch while the difference is less than $1 / 10$ in the patch with a radius of 50 . However, this does not imply that evolution at the edge and in the centre are completely independent. Given the values for $G_{d}$ which did evolve, the direct effect of loosing offspring is limited to cells near the border of the patch. Nonetheless, compared to area A1 a reduction in dispersal distance is still noticeable in ring A2, (Figure 4), i.e. in a distance which is about seven times further away from the edge than the mean value for $G_{d}$ in the patch's centre. Apparently, gene flow "transfers" the selective pressure on $G_{d}$ near the patch's edge far into the interior of the patches.

The simulation results clearly demonstrate the existence of a minimum patch area necessary for the evolution of locally adapted dispersal strategies. If we assume the average diameter of a tree crown to be $5 \mathrm{~m}$, the minimal area of a (circular) patch in which we could expect to find a gradient in dispersal strategy would be about $0.2 \mathrm{~km}^{2}$ (500 m diameter). In contrast, for small herbs with a average diameter of only $10 \mathrm{~cm}$, a spatial structure could eventually develop in a patch of only $75 \mathrm{~m}^{2}$.

The evolution of dispersal distance and consequently the size of the values predicted above would certainly be different if model parameters were altered or new ones added. For example, an increase in the longevity of organisms, trade-offs between dispersal distance and competitiveness (cf. Greene and Johnson 1993; Geritz 1995), or habitat disturbance (cf. Comins 1982; Gandon and Michalakis 2001) would presumably all alter the evolution of dispersal distance and the emergence of spatial patterns.

From experimental work and field-observations we know that a variety of plant species is able to adapt their propagation strategy to selective pressures. Michaels et al. (1988) confirmed that intraspecific adaptations of dispersal strategies are generally possible. More specifically, a plant can influence the dispersal capabilities of its offspring because seed sizes of individual plants vary (Geritz 1995). An example for a direct adaptation to 
317 landscape structure is the change of the reproductive mode from propagation via seeds 318 (long distance dispersal) to vegetative (nearest neighbour dispersal) depending on local 319 circumstances. Prati and Schmid (2000) discovered that Ranunculus reptans invested 320 heavily into sexual reproduction when close to water, but more into vegetative reproduc321 tion on land. In this case the differentiation is only an indirect adjustment to landscape 322 structure, as it results from a difference in the intensity of competition with a low chance 323 of establishment from seeds away from water. Kanno and Seiwa (2004) report that the 324 reproductive mode (sexual or vegetative) of the clonal forest understorey shrub Hydrangea 325 paniculata is influenced by small scale variations in the dynamics of canopy trees. These 326 findings, as well as our simulation results, prove that adjustments of dispersal strategies 327 to local demographic situations are in principle possible and are selected for under certain 328 conditions.

329

Acknowledgements. We are grateful for the financial support by the German Science 331 Foundation (DFG PO 244/3-1). 


\section{${ }_{332}$ References}

Appelt, M. and Poethke, H. J. 1997. Metapopulation dynamics in a regional population of the blue-winged grasshopper (Oedipoda caerulescens; Linnaeus, 1758). - J. Insect Conserv. 1: 205-214.

Berec, L. 2002. Techniques of spatially explicit individual-based models: construction, simulation, and mean-field analysis. - Ecol. Model. 150: 55-81.

Bleher, B., Oberrath, R. and Böhning-Gaese, K. 2002. Seed dispersal, breeding system, tree density, and the spatial pattern of trees: A simulation approach. - Basic Appl. Ecol. 3(2): 115-123.

Bullock, J. M. and Clarke, R. T. 2000. Long distance seed dispersal by wind: measuring and modelling the tail of the curve. - Oecologia 124: 506-521.

Case, T. J. and Taper, M. L. 2000. Interspecific competition, environmental gradients, gene flow, and the coevolution of species borders. - Am. Nat. 155: 583-605.

Clark, J. S., Silman, M., Kern, R., Macklin, E. and HilleRisLambers, J. 1999. Seed dispersal near and far: patterns across temperate and tropical forests. - Ecology 80(5): $1475-1494$.

Comins, H. N. 1982. Evolutionarily stable strategies for localized dispersal in two dimensions. - J. Theor. Biol. 94: 579-606.

Comins, H. N., Hamilton, W. D. and May, R. M. 1980. Evolutionarily stable dispersal strategies. - J. Theor. Biol. 82: 205-230.

Dytham, C. 2003. How landscapes affect the evolution of dispersal behaviour in reef fishes: results from an individual-based model. - J. Fish Biol. 63(Supplement A): 213-225.

Ennos, R. A. 2001. Inference about spatial processes in plant populations from the analysis 
of molecular markers. - In: Silvertown, J. and Antonovics, J. (eds.), Integrating ecology and evolution in a spatial context. Blackwell Science, pp. 45-71.

Ezoe, H. 1998. Optimal dispersal range and seed size in a stable environment. - J. Theor. Biol. 190: 287-293.

Frank, S. A. 1986. Dispersal polymorphisms in subdivided populations. - J. Theor. Biol. 122: 303-309.

Gandon, S. and Michalakis, Y. 2001. Multiple causes of the evolution of dispersal. - In: Clobert, J., Danchin, E., Dhondt, A. A. and Nichols, J. D. (eds.), Dispersal. Oxford University Press, pp. 155-167.

Geritz, S. A. H. 1995. Evolutionarily stable seed polymorphism and small-scale spatial variation in seedling density. - Am. Nat. 146(5): 685-707.

Greene, D. and Johnson, E. A. 1993. Seed mass and dispersal capacity in wind-dispersed diaspores. - Oikos 67: 69-74.

Haldane, J. B. S. 1956. The relation between density regulation and natural selection.Proc. R. Soc. Lond., Ser. B: Biol. Sci. 145: 306-308.

Hamilton, W. D. and May, R. M. 1977. Dispersal in stable habitats. - Nature 269: $578-581$.

Hanski, I. 1994. A practical model of metapopulation dynamics. - J. Anim. Ecol. 63: $151-162$.

Hanski, I., Erälahti, C., Kankare, M., Ovaskainen, O. and Sirén, H. 2004. Variation in migration propensity among individuals maintained by landscape structure. - Ecol. Lett. 7: 958-966.

Hastings, A. 1983. Can spatial variation alone lead to selection for dispersal?. - Theor. Popul. Biol. 24: 244-251. 
Hovestadt, T., Messner, S. and Poethke, H. J. 2001. Evolution of reduced dispersal mortality and 'fat-tailed' dispersal kernels in autocorrelated landscapes. - Proc. R. Soc. Lond., Ser. B: Biol. Sci. 268: 385-391.

Hovestadt, T., Poethke, H. J. and Messner, S. 2000. Variability in dispersal distances generates typical successional patterns: a simple simulation model. - Oikos 90(3): 612619.

Kanno, H. and Seiwa, K. 2004. Sexual vs. vegetative reproduction in relation to forest dynamics in the understorey shrub, Hydrangea paniculata (Saxifragaceae). - Plant Ecol. 170: $43-53$.

Kasuya, E. 2000. Kin-biased dipsersal behaviour in the mango shield scale, Milviscutulus mangiferae. - Anim. Behav. 59: 629-632.

Kisdi, E. 2004. Conditional dispersal under kin competition: extension of the HamiltonMay model to brood size-dependent dispersal. - Theor. Popul. Biol. 66: 369-380.

Lambin, X., Aars, J. and Piertney, S. B. 2001. Dispersal, intraspecific competition, kin competition and kin facilitation: a review of the empirical evidence. - In: Clobert, J., Danchin, E., Dhondt, A. A. and Nichols, J. D. (eds.), Dispersal. Oxford University Press, New York, pp. 110-122.

Levin, S. A., Cohen, D. and Hastings, A. 1984. Dispersal strategies in patchy environments. - Theor. Popul. Biol. 26: 165-191.

Levin, S. A., Muller-Landau, H. C., Nathan, R. and Chave, J. 2003. The ecology and evolution of seed dispersal: a theoretical perspective. - Annu. Rev. Ecol. Syst. 34: $575-604$.

Levin, S. A. and Pacala, S. W. 1997. Theories of simplification and scaling of spatially distributed processes. - In: Tilman, D. and Kareiva, P. (eds.), Spatial ecology: the role 
of space in population dynamics and interspecific interactions. Princeton University Press, pp. 271-295.

Metz, J. A. J. and Gyllenberg, M. 2001. How should we define fitness in structured metapopulation models? Including an application to the calculation of evolutionarily stable dispersal strategies. - Proc. R. Soc. Lond., Ser. B: Biol. Sci. 268: 499-508.

Michaels, H. J., Benner, B., Hartgerink, A. P., Lee, T. D., Rice, S., Willson, M. F. and Bertin, R. I. 1988. Seed size variation: magnitude, distribution, and ecological correlates. - Evol. Ecol. 2: 157-166.

Motro, U. 1991. Avoiding Inbreeding and Sibling Competition: The Evolution of Sexual Dimorphism for Dispersal. - The American Naturalist 137(1): 108-115.

Murrell, D. J., Travis, J. M. J. and Dytham, C. 2002. The evolution of dispersal distance in spatially-structured populations. - Oikos 97(2): 229-236.

Nathan, R., Safriel, U. N., Noy-Meir, I. and Schiller, G. 2000. Spatiotemporal variation in seed dispersal and recruitment near and far from Pinus halepensis trees. - Ecology 81(8): 2156-2169.

Poethke, H. J. and Hovestadt, T. 2002. Evolution of density- and patch-size-dependent dispersal rates. - Proc. R. Soc. Lond., Ser. B: Biol. Sci. 269: 637-645.

Prati, D. and Schmid, B. 2000. Genetic differentiation of life-history traits within populations of the clonal plant Ranunculus reptans. - Oikos 90(3): 442-456.

Rousset, F. and Gandon, S. 2002. Evolution of the distribution of dispersal distance under distance-dependent cost of dispersal. - J. Evol. Biol. 15: 515-523.

Streiff, R., Ducousso, A., Lexer, C., Steinkellner, H., Gloessl, J. and Kremer, A. 1999. Pollen dispersal inferred from paternity analysis in a mixed oak stand of Querkus robur L. and Q. petraea (Matt.) Liebl. - Mol. Ecol. 8(5): 831-841. 
${ }_{427}$ Tilman, D., Lehman, C. L. and Kareiva, P. 1997. Population dynamics in spatial habi${ }^{428}$ tats. - In: Tilman, D. and Kareiva, P. (eds.), Spatial ecology: the role of space in 429 population dynamics and interspecific interactions. Princeton University Press, pp. 3$430 \quad 20$.

${ }_{431}$ Travis, J. M. and Dytham, C. 1999. Habitat persistence, habitat availability and the ${ }^{432}$ evolution of dispersal. - Proc. R. Soc. Lond., Ser. B: Biol. Sci. 266: 723-728.

${ }_{433}$ Yao, J., Holt, R. D., Rich, P. M. and Marshall, W. S. 1999. Woody plant colonization in ${ }_{434}$ an experimentally fragmented landscape. - Ecography 22(6): 715-728. 


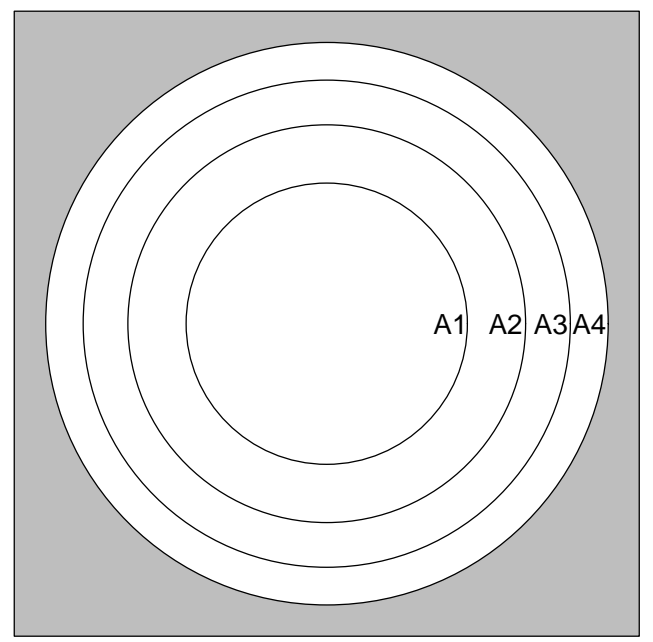

Figure 1: Simple patch-matrix landscape used in simulations. (white $=$ habitat, gray $=$ matrix $). \quad$ The indices mark the evaluation areas of approximately equal carrying capacity seperately analysed for emergent dispersal strategies. Simulations were run on patches with radii varying from 25 to 150 in steps of 25 . 

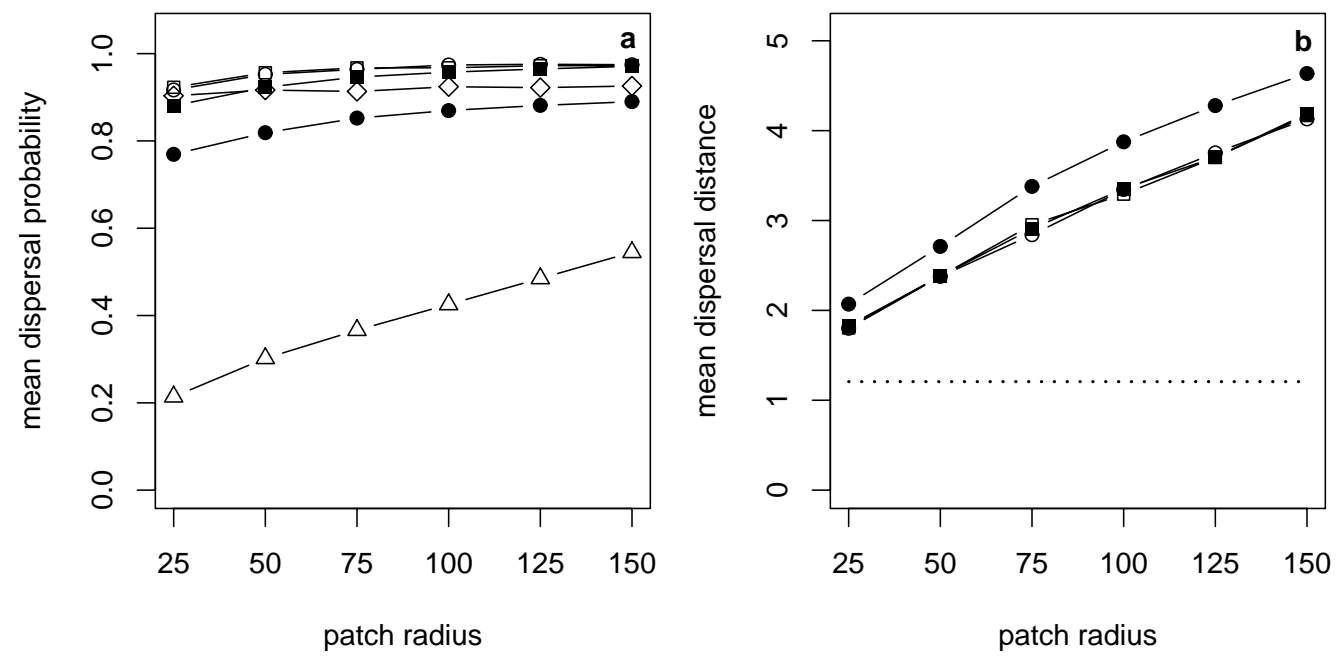

Figure 2: (a) Evolved mean dispersal probabilities (except for NE and S, parameter $G_{p}$ ) with either kernel NE + (open circles), kernel NE (filled circles), $\mathrm{S}+$ (open squares), $\mathrm{S}$ (filled squares), NN (open diamonds), or G (open triangles) in different patch sizes. For kernels NE and $\mathrm{S}$ the evolved mean dispersal probability is only the emergent consequence of selection on trait $G_{d}$. (b): Evolved mean dispersal distances (parameter $G_{d}$ ) either for kernel $\mathrm{NE}+$ (open circles), NE (filled circles), $\mathrm{S}+$ (open squares), or $\mathrm{S}$ (filled squares) in different habitat patch sizes. Dotted line indicates mean dispersal distance for nearest neighbour dispersal. The plotted trait values are averages of the traits of all individuals in a patch. 

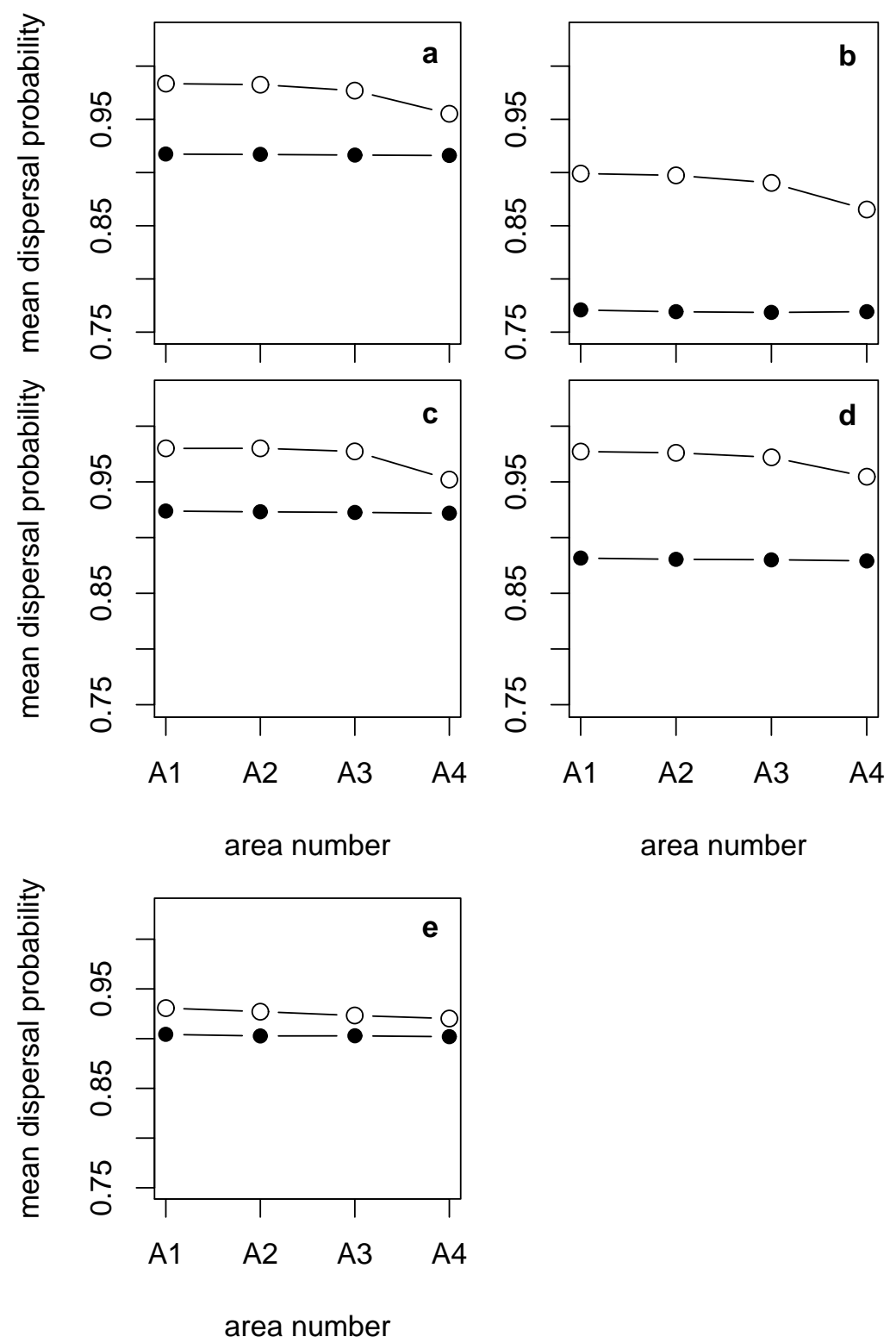

area number

Figure 3: Distribution of mean dispersal probability (except for NE and S, parameter $G_{p}$ ) across the different patch zones outlined in Figure 1 either for kernel NE+ (a), kernel NE (b), kernel S+ (c), kernel S (d), or NN (e). Open circles: patch-radius 150, filled circles: 25 cells. For kernel NE and $\mathrm{S} G_{p}$ as such is not an evolving trait but rather the fraction of seeds leaving the home cell given the evolved mean dispersal distance $\overline{G_{d}}$. The plotted trait values result from seperate averaging of individuals within the evaluation zones A1 to A4 (Figure $1)$. 

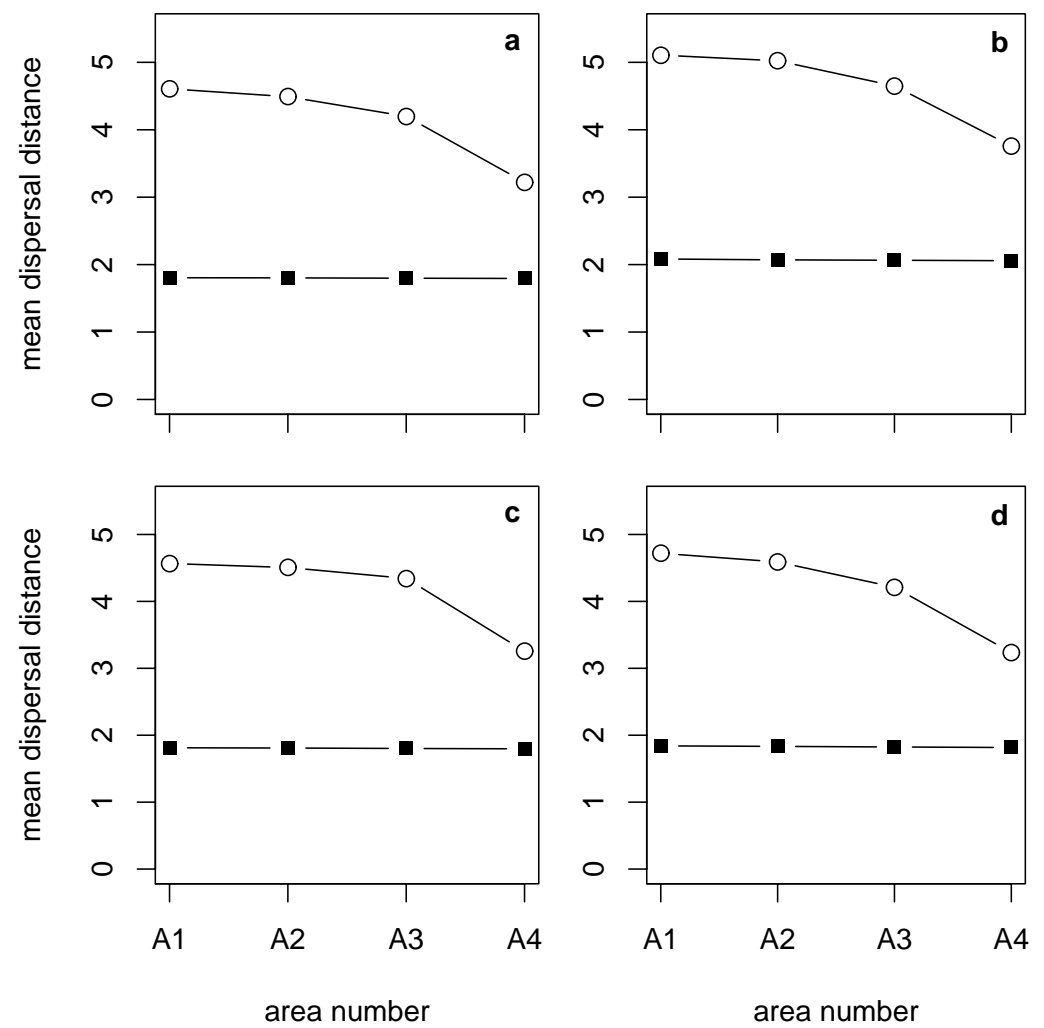

Figure 4: Distribution of mean dispersal distance with kernel NE+ (a), NE (b), S+ (c) or $\mathrm{S}(\mathrm{d})$. The panels show mean dispersal distances in evaluation areas as stated in Figures 1. Open circles: patch-radius 150, filled squares: 25 cells. The plotted trait values result from seperate averaging of individuals within the evaluation zones A1 to A4 (Figure 1). 

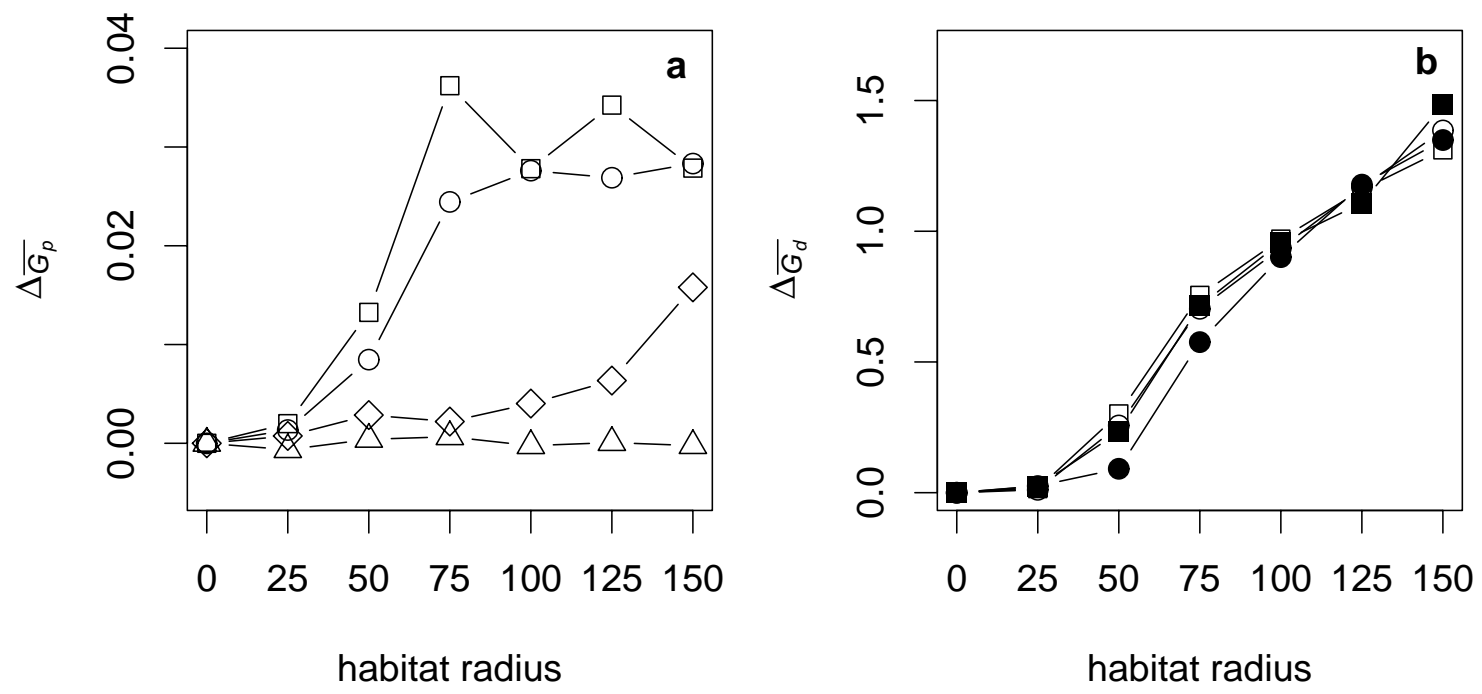

Figure 5: Difference of evolved mean dispersal probability $\left(\overline{G_{p}}\right)$ and mean dispersal distance $\left(\overline{G_{d}}\right)$ between area $\mathrm{A} 1$ and $\mathrm{A} 4$ in relation to habitat radius. (a) Mean dispersal probability $\left(\overline{G_{p}}\right)$ and (b) mean $1 / \alpha$ in kernels $\mathrm{NE}$ and $\mathrm{NE}+$ and mean $2 / \alpha$ in kernels $\mathrm{S}$ and $\mathrm{S}+\left(\overline{G_{d}}\right)$. Open circles: kernel $\mathrm{NE}+$, filled circles: kernel $\mathrm{NE}$, open squares: kernel $\mathrm{S}+$, filled squares: kernel S, open diamonds: kernel NN, open triangles: kernel G.
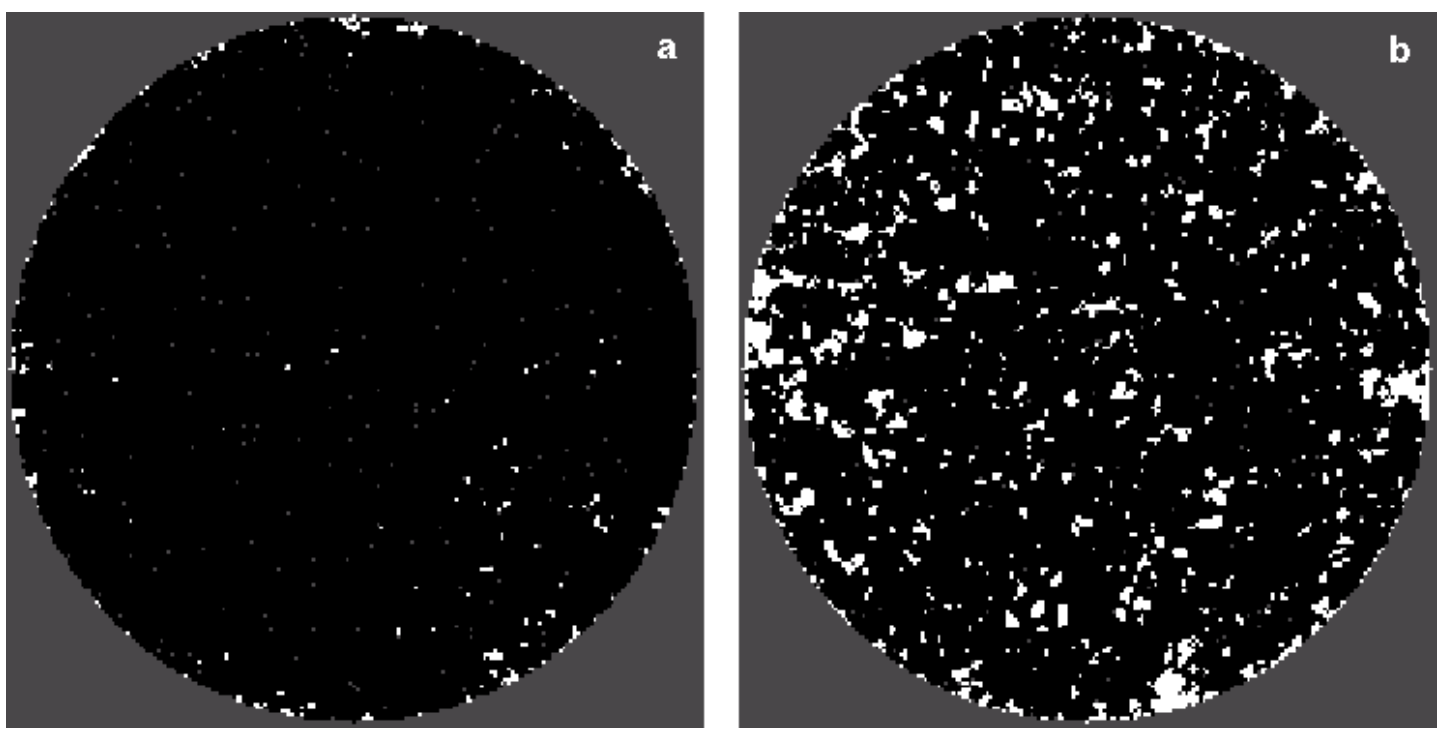

Figure 6: Similarity of $G_{p}$-values in patches of radius 100 with either kernel NE+ (a) or kernel NN (b) at the end of a simulation run. Occupied habitat cells are coloured either black or white. A cell is coloured white, if it is surrounded by at least seven cells with same trait values or matrix cells. Dark gray areas outside patch boundary indicate matrix, inside the patch currently empty cells. In contrast to our other findings here we find different pictures with different mutation rates. 


\begin{tabular}{|c|c|c|}
\hline kernel & distance probability density & evolving traits \\
\hline Global (G) & 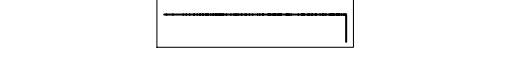 & $G_{p}$ \\
\hline Nearest Neighbour (NN) & & $G_{p}$ \\
\hline Negative Exponential (NE) & & $G_{d}$ \\
\hline Negative Exponential plus disp. probability $(\mathrm{NE}+)$ & & $G_{d}, G_{p}$ \\
\hline Skewed (S) & & $G_{d}$ \\
\hline Skewed plus disp. probability $(\mathrm{S}+)$ & & $G_{d}, G_{p}$ \\
\hline
\end{tabular}

Table 1: Pictographic presentation of the different dispersal kernels implemented in simulation models (for more details see text). The term "plus disp. probability" means that these kernels make use of both genetic characters, dispersal distance and dispersal probability. 